ISSN: 2600-5859

\title{
Manejo de actividades lúdicas con estudiantes de medicina en inglés
}

\author{
Management of ludic activities with medical students in English \\ Christian Eduardo Meza Fiallos. ${ }^{1}$
}

\section{Recibido:01-12-2019 / Revisado: 02-01-2020 /Aceptado: 18-01-2020/ Publicado: 07-02-2020}

Resumen

DOI: $\underline{\text { https://doi.org/10.33262/concienciadigital.v3i1.1.1140 }}$

En la actualidad, las clases impartidas en la carrera de medicina siguen manteniendo su estilo y forma tradicional que por décadas ha puesto al docente como agente principal en el proceso de enseñanza aprendizaje quedando el alumno relegado a un participante pasivo en su formación de aprendizaje, razón por la cual es imperioso la aplicación de actividades lúdicas y entretenidas y más importante aun cuando se pretende enseñar en una segunda lengua como es el inglés. Objetivo: Mejorar la forma de enseñanza del inglés sobre temas médicos a través del uso de actividades lúdicas y activas que despierten el interés en los alumnos por aprender medicina en una segunda lengua. Método: Se realizó una intervención educativa a 2 grupos de estudiantes de cuarto semestre de la carrera de medicina de la Escuela Superior Politécnica de Chimborazo mediante una prueba que permita detectar el rendimiento y nivel de captación del tema del primer grupo recibiendo la clase de manera tradicional para después analizar los datos y contrastarlos con la prueba del segundo grupo recibiendo las clases con actividades dinámicas y lúdicas que permitan evidenciar un aumento significativo de la comprensión de los temas. Se tabularon los datos y se presentaron a los alumnos para su posterior discusión. Resultados: Se puede notar claramente una mejora en la atención, interés y comprensión de los temas médicos impartidos en inglés por parte de los alumnos y consecuentemente mejora su retención. Conclusiones: Con la presente intervención educativa aplicada en el campo médico con el idioma inglés se logró determinar la importancia de usar estrategias metodológicas dinámicas en las clases debido a la gran influencia que tiene en la atención, retención y comprensión en los alumnos.

Palabras claves: Actividades lúdicas, mejora en la atención, interés, estudiantes de medicina

${ }^{1}$ Escuela Superior Politécnica de Chimborazo, Riobamba, Ecuador, christian.meza@espoch.edu.ec 
ISSN: 2600-5859

\begin{abstract}
At present, the classes taught in the career of medicine continue to maintain their traditional style and form that for decades has put the teacher as the main agent in the teaching-learning process, leaving the student relegated to a passive participant in their learning process, reason for which it is imperative the application of fun and entertaining activities and more importantly when it is intended to teach in a second language such as English. Objective: To improve the way of English teaching on medical subjects through the use of fun and active activities that arouse interest in students to learn medicine in a second language. Method: An educational intervention was carried out on 2 groups of fourth semester students of the career of medicine of Escuela Superior Polytechnical de Chimborazo by means of a test that allows to detect the performance and level of retention of the subject of the first group receiving the class in a traditional way to later analyze the data and contrast it with the test of the second group receiving the classes with dynamic and fun activities that allow to demonstrate a significant increase in the understanding of the topics. Data were tabulated and presented to students for later discussion. Results: An improvement in the attention and interest of the medical subjects taught in English on part of the students can be clearly noticed and consequently improves their retention. Conclusions: With the present educational intervention applied in the medical field with the English language, it was possible to determine the importance of using dynamic methodological strategies in the classes due to the great influence it has on attention, retention and understanding in students.
\end{abstract}

Keywords: Ludic activities, improvement in retention, interest, students of medicine

\title{
Introducción
}

En nuestra época actual ya nadie discute la importancia del inglés en el campo académico y en el campo laboral a nivel mundial. El campo de la medicina no puede ser la excepción, gran cantidad de libros, artículos científicos e investigaciones están en inglés lo cual obliga a profesores, estudiantes y profesionales de la salud a adaptarse a esta tendencia y prepararse mejor para comprender este idioma. También es importante por la necesidad de viajar a otro país por trabajo, o por participar en congresos científicos donde hay intercambio de conocimiento y el inglés es el medio por el cual los participantes de los diferentes países se comunican. 
Debido a tan grande importancia, es necesario mejorar el enfoque o metodología empleado en las clases de medicina en inglés para hacer que estos temas queden bien aprendidos en esta segunda lengua y puedan ser utilizado en la práctica diaria profesional de cada médico dentro y fuera del país. La mejor estrategia educativa para aprender algo nuevo siempre han sido los juegos porque puede levantar a los alumnos de sus pupitres y los motiva a realizar un proceso de aprendizaje a través del movimiento.

Sin embargo, si se analiza el rol que han tenido los juegos durante la historia del hombre aquí en la tierra, encontramos que es muy antigua pero lastimosamente todavía no se le otorga un valor educativo y no se le da prioridad y mucha importancia en las aulas. El ser humano aprende jugando y se divierte haciéndolo. Cuando se utiliza juegos didácticos, lúdicos en clase, el alumno deja de lado su consciencia de que está aprendiendo para entregarse a un ambiente amigable y ameno donde se practica y refuerza el conocimiento aprendido en clase.

Es penoso, pero aun en las clases de la universidad, se mantiene el estilo rígido y tradicional de clases expositivas donde el alumno es un mero oidor, receptor del conocimiento y no un actor activo que construya el conocimiento. Aunque si se está teniendo un cambio positivo sobre la importancia del uso de juegos que refuercen el tema de la clase, falta mucho camino por recorrer.

De ahí la importancia de irse por una educación que promueva el involucramiento, participación activa y creatividad del alumno a través de los juegos y actividades lúdicas.

En las clases de medicina no ha cambiado mucho la forma de impartir la asignatura, aún se mantiene las clases magistrales en el aula de clase, en el laboratorio ya sea con videos o exposiciones pero se ha encontrado que esto estimula poco al estudiante ya que llega a convertirlo en sujeto pasivo al escuchar las explicaciones de los temas desde su asiento, de ahí la importancia de aplicar estrategias metodológicas que permitan a los alumnos interactuar de manera dinámica, divertida y lúdica. Siempre se dice que el aprendizaje en las aulas debe ser asimilado utilizando la teoría, la práctica y el método como un todo para que el aprendizaje sea significativo. Por esa razón, las explicaciones y el aprendizaje en si se enriquece mucho más cuando se mejora el método, es decir cuando se da atención al cómo dar las clases. 
ISSN: 2600-5859

Una de las tantas estrategias que se pueden utilizar en las clases de inglés en medicina son los mapas conceptuales, juegos mentales y el aprendizaje basado en problemas "Problem-Based learning". Vallejos, Latorre y Ávila (2016) menciona que esto, "permite la discusión constructiva entre estudiantes, estimulan el razonamiento para resolución de problemas y la generación de propuestas en el marco del aprendizaje autodirigido" (p 100) para lo cual pueden formar grupos de trabajo, dejar sus bancas, pararse, moverse con libertad sin que exista esa tensión e incomodidad de estar horas sentado recibiendo y anotando información y así se cambia el enfoque con el cual se da las clases al pasar de conductismo al enfoque constructivista y enfoque comunicativo donde al alumno se lo involucra de manera activa y se le despierta la conciencia sobre para que le sirve lo que aprende y como hacer un buen uso del idioma.

Por ejemplo, se puede formar grupos de trabajo en donde los alumnos tengan que resumir las ideas principales de lecturas de casos clínicos con la ayuda de mapas conceptuales para que sea más fácil identificar los síntomas de la enfermedad y puedan encontrar el mejor tratamiento de la misma; de esta manera, los alumnos se involucrarán de manera más activa en la consecución del problema.

García (2000), menciona que el elemento lúdico de los juegos "ayuda considerablemente a desinhibir e incrementar la participación del alumno, sobre todo la participación creativa" (p 123). así de una forma relajada y entretenida aprenden nuevos conocimientos o refuerzan los aprendidos. Además de los juegos mencionados previamente, hay otras herramientas que se las pueden utilizar por su alto contenido lúdico y participativo a los alumnos. Pimienta (2012) sugiere algunas estrategias metodológicas para educación superior una de ellas es el Debate y menciona que "es una competencia intelectual que debe efectuarse en un clima de libertad, tolerancia y disciplina" (p 109).

El profesor escoge el tema de medicina, asigna un moderador y le ayuda a desarrollar un guión en inglés para que presente el tema y señale los puntos a discutir y el objetivo del debate. El debate puede ser sobre que tratamientos son menos invasivos y dolorosos para el paciente de cáncer y tiene como finalidad hacer que los alumnos puedan de manera libre y espontánea defender una 
ISSN: 2600-5859

idea y hacer replicar. Para lo cual se les da tiempo para que puedan prepararlo con anticipación por tratarse de una lengua extranjera.

Otra actividad menciona por Pimienta (2012) es el uso del taller donde los alumnos en grupo "aplican los conocimientos adquiridos en una tarea específica, generando un producto que es resultado de la aportación de cada uno de los miembros del equipo" (p 109).

Por ejemplo, se puede hacer una actividad grupal a modo de taller donde se pueda aplicar un juego llamado "Snap in Passive voice". Entonces, después de dar la explicación de la voz pasiva y activa en inglés a los alumnos se les pide que hagan 10 tarjetas del tamaño de la cuarta parte de una hoja A4 de papel bond, donde tendrán que escribir 5 oraciones en voz activa sobre el sistema circulatorio, una oración en cada una de las tarjetas, para después transformarlas en voz pasiva y escribirlas en las demás tarjetas igualmente una oración en cada tarjeta.

Tabla 1. Active Voice

\begin{tabular}{|c|c|}
\hline Active Voice & Passive Voice \\
\hline $\begin{array}{l}\text { The blood carries the nutrients and the } \\
\text { throughout the body }\end{array}$ & $\begin{array}{l}\text { exygen The nutrients and the oxygen are carried by the blood } \\
\text { throughout the body. }\end{array}$ \\
\hline receives the blo & om the $\mathrm{T}$ \\
\hline ody and sent it to & पात \\
\hline
\end{tabular}

Fuente: https://accessmedicina. mhmedical.com/content aspx ?bookid=1506\&sectionid=98183303

Elaborado por: El autor

Una vez que los alumnos han terminado, se forman grupos de 4 estudiantes, se mezclan las tarjetas y se ponen boca bajo formando una pila, los alumnos se paran y se ubican alrededor del pupitre y cada uno levanta una tarjeta y la deja boca arriba para que todos puedan leerla. Cuando las 2 tarjetas con las oraciones del mismo mensaje una en voz activa y la otra en voz pasiva aparezcan, el primero que diga "Snap" se lleva el par. Gana el que tenga más tarjetas cuando se ha terminado la pila.

Otro ejemplo de un ejemplo dinámico y activo es "Es o no es". El objetivo es practicar las patologías más importantes que estudian en el semestre, por ejemplo, hipertensión, infarto, amigdalitis, neumonía, etc. 
Procedimiento:

1. El profesor imprime en tarjetas las definiciones de las patologías en inglés

2. Se forman 2 grupos

3. El profesor escoge a un participante de cada equipo para iniciar el juego

4. Se hace un sorteo y el alumno que salga escoge la tarjeta.

5. El profesor lee la tarjeta, si la definición es correcta, el alumno deberá repetirla con sus propias palabras practicando de esta manera la destreza de hablar "Speaking skill". Si la definición fuera incorrecta, el alumno debe quedarse callado y gana un punto.

6. Si el alumno se queda callada cuando la definición es correcta, se pasa el turno al otro alumno y el primero pierde el punto.

7. El equipo que tenga más puntos gana.

Otro juego para practicar vocabulario concerniente a los servicios y departamentos en el hospital y desarrollar la destreza de "Speaking" es "At the hospital". Se debe hacer un tablero, tarjetas con el nombre de los servicios y departamentos y una bolsa donde estarán las tarjetas con los nombres de los servicios y los departamentos.

Procedimiento:

1. En el tablero se pondrán fotografías de los servicios y departamentos del hospital como, por ejemplo: Operating rooms and sterilization, cardiology, nursing department, intensive care unit, etc.

2. Se forman dos grupos

3. Se ponen las tarjetas en la bolsa

4. Cada equipo elige un participante y pasa al frente

5. Cada participante coge 3 fichas de la bolsa e intenta encontrar la fotografía que dice la tarjeta. Después debe decir los nombres de los departamentos y decir que se les hace a los pacientes ahí. Si el primero encuentra 3 parejas y puede decir que se les hace a los pacientes ahí, gana un punto. Después se continua con el otro estudiante y gana el juego el grupo que acumule más puntos.

El resultado e involucramiento de los alumnos en las actividades es fantástico y se puede notar claramente en su aprendizaje. Cuando los alumnos están en contacto con el tema teórico de medicina, no importa si es en inglés, ellos de igual forma se esfuerzan y hablan en inglés debido 
ISSN: 2600-5859

al ambiente divertido que general la competición y el juego. Lightbown \& Spada (2011) menciona en su libro que se han hecho investigaciones sobre cómo se adquiere el idioma cuando los alumnos colaboran e interactúan mutuamente en el uso del idioma y han llegado a la conclusión de que el hecho que el alumno tenga que producir el idioma hablándolo en contextos realistas lo empuja para procesar el idioma de manera más profunda.

Con respecto a esto, Swain (1997) menciona que al prepararse para hablar o escribir, los alumnos deben poner más atención al significado que tienen que expresar más bien que a la comprensión del idioma, lo cual por supuesto tiene que ser entendido. Es decir, que a través de las actividades entre los alumnos donde colaboran, compiten e interactúan para alcanzar un objetivo común les motivan para no solo entender el tema sino expresarlo con sus propias palabras.

Rosas (2015) menciona que la utilidad del juego en contextos educativos influye en el logro de un ambiente de evaluación invisible que genera mayor bienestar y permite superar la tensión y el aburrimiento producido pruebas tradicionales.

Con respecto a esto, Lee W. R. (2008) menciona que los juegos son agradables y divertidos y que en esencia cumplen el propósito de superar el desempeño del resto o de uno mismo de manera amigable y motivante. Así, los diferentes estudios de los juegos en contextos educativos ayudan sobremanera a superar obstáculos, motivar a los alumnos y ayudarles a desarrollar mejor su potencial en el aprendizaje de una lengua.

Sin embargo, en el caso de las clases de inglés enfocadas a temas médicos no se ha avanzado mucho en el uso de juegos o actividades que les levanten a los alumnos y les obliguen a participar de manera más activa y atrayente.

De ahí la necesidad de la presente intervención educativa para evaluar de manera objetiva el impacto de los juegos en clases de medicina en inglés y si existe una diferencia significativa en la comprensión general en los alumnos.

\section{Metodología}

El presente trabajo investigativo se lo realizo con el método cuantitativo con los estudiantes de cuarto semestre de la carrera de medicina de la Escuela Superior Politécnica de Chimborazo durante los meses de noviembre y diciembre de 2019 con una población de 70 de estudiantes a 
ISSN: 2600-5859

quienes se aplicó una evaluación escrita sobre un caso clínico donde aparezcan los temas gramaticales como la voz pasiva "Passive Voice", los departamentos y servicios que dan los hospitales y patologías como neumonía, infartos entro otros.

La prueba tenía unas 30 preguntas sobre temas específicos sobre patologías comunes en pacientes con neumonía y sus respectivos departamentos del hospital. Primero se abordó y analizo esta lectura a través de preguntas y respuestas, se socializo el vocabulario nuevo con los alumnos y después se administró una prueba para evaluar su comprensión general del tema.

Posteriormente se calificó la prueba y se determinó la media del primer grupo para saber cuantitativamente cuanto habían entendido y dominado el tema para luego contrastar con los resultados del segundo grupo con quien se abordó el análisis de la lectura con otro enfoque, se aisló las palabras difíciles, se hizo la explicación y su refuerzo con el juego "Snap”, se aprendió y practicó los departamentos con sus síntomas con el tablero y las tarjetas que indican que se le hacía al paciente ahí.

Se calificó la prueba del segundo grupo y se calculó su media aritmética para saber su nivel de captación y comprensión del tema. Después se hizo una encuesta sobre las preferencias de los alumnos al recibir las clases de inglés de medicina para saber la importancia que le daban a los juegos comparando su comprensión y ambiente de la clase.

Así se pueda determinar si el uso de juegos que se espera motive, despierten el interés y les estimulen a aprender tienen un impacto positivo en su aprendizaje de los alumnos.

\section{Resultados}

Habiendo realizado el conteo y tabulación de los datos, se obtuvieron los siguientes resultados de las dos pruebas y con los 2 grupos de estudiantes.

Tabla 2. Media de los 2 grupos sin y con enseñanza a través de juegos y dinámicas

\begin{tabular}{lcc}
\hline & Primer Grupo & Segundo Grupo \\
\hline Clases impartidas con juegos lúdicos & $60,78 \%$ & \\
Clases impartidas con juegos lúdicos & & $89,40 \%$ \\
\hline
\end{tabular}

Fuente: Test de los estudiantes

Elaborado por: El autor 
ISSN: 2600-5859

Como se muestra en la tabla 2, hay un contraste marcado con la media de las calificaciones alcanzadas por el primer grupo y segundo grupo antes y después de la intervención. Si se analiza los porcentajes sobre 100\%, el primer grupo alcanzó una media alta de 60,78\% en el test de comprensión de conocimientos sobre el caso clínico sobre "Cough and fever for four days" con el cual no se usó los juegos para abordar la lectura. Esto sugiere la dificultad por parte de los alumnos para comprender bien el caso clínico al no entender bien los síntomas del paciente y su tratamiento. Mientras que el segundo grupo alcanzó una media alta de 89,40\% con respecto al primero después de haber hecho una explicación previa sobre la voz pasiva y su práctica posterior a través del juego "Snap" explicado previamente. También se identificó las palabras difíciles para los alumnos que tenían relación con los síntomas, se explicó su significado y se reforzó su significado con el juego "Bingo" donde los alumnos tenían que encontrar los significados en español en el tablero de Bingo. También se tomó los síntomas y afecciones respiratorias del paciente y se hizo un juego donde los alumnos tenían que decir si los síntomas escritos en la tarjeta correspondían con los síntomas del paciente. Los resultados arrojados por el test al segundo grupo indica que el enfoque lúdico utilizado en la clase logro que los alumnos entiendan y capten mejor los aspectos relacionados con este caso clínico de neumonía.

Tabla 3.- Encuesta realizada a los alumnos sobre el uso de los juegos.

\begin{tabular}{lcc}
\hline Encuesta & $\mathrm{Si}$ & No \\
\hline Considera que se puede entender más con el juego & $95 \%$ & $5 \%$ \\
lúdico. & & $10 \%$ \\
Prefiere recibir clase con juegos. & $90 \%$ & $6,79 \%$ \\
Qué porcentaje de importancia les da a los juegos en & $93,21 \%$ & \\
la clase de inglés de medicina. (del 1 al 100) & &
\end{tabular}

Fuente: Test de los estudiantes

Elaborado por: El autor

En tabla 3 se puede ver el grado de importancia y preferencia que los alumnos dan a clases de reforzamiento de inglés en medicina basado en su nivel captación y comprensión del tema tratado en clase. Es interesante notar que de toda la población el 95\% de los encuestados dijo haber entendido más que con una clase tradicional de leer, analizar y contestar preguntas. 
ISSN: 2600-5859

Sin embargo, el 5\% mencionó que al hacer un juego se pierde tiempo valioso al no dedicar más tiempo a la lectura y al análisis de los casos clínicos debido a la complejidad del tema. Esto deja ver simplemente que el nivel de inglés de dichos alumnos es alto y por dicha razón el alumno no necesita reforzar los aspectos característicos del idioma inglés como la gramática y vocabulario para entender los temas de medicina.

Adicionalmente, se les preguntó a los alumnos si preferirían recibir las clases de inglés con juegos y el $90 \%$ dijo que sí debido a que les resulta más instructivo y entretenido. A través de las encuestas, mencionaron que entienden mejor los temas de gramática porque el juego les obliga a saberse y memorizarse las reglas de gramática, en este caso la voz pasiva y el deletreo de las palabras concernientes a medicina en inglés. En contraste, el 10\% de los alumnos mencionaron que no porque se podría abordar la clase también con diapositivas y videos y no solamente con juegos.

Finalmente, se les preguntó a los encuestados qué importancia le daban a los juegos de manera didáctica y la gran mayoría con un 93,21\% mencionó que mucha debida a que están más activos y atentos a las explicaciones del tema y posterior aplicación en el juego; de esta manera pueden reforzar el tema para su mejor comprensión. Mientras el 6,79\% dijo no darle mucha importancia por tratarse de clases de medicina y se necesita más tiempo para dedicarse a esos temas.

De ahí que Herrell \& Jordan (2012) mencionen que una exposición del tema que tenga significado para los alumnos no es suficiente para la adquisición del idioma sino más bien las muchas oportunidades que tengan los alumnos para la interacción del idioma. Esto se consigue muy bien con los juegos ya que les da razones para comunicarse y perfeccionar la producción del lenguaje.

\section{Discusión}

A través de la presente investigación se ha comprobado que los juegos añaden un valor intrínseco a las clases en general. Independientemente si es en inglés o en español lo juegos permiten involucrar a los alumnos de manera activa y cooperativa hacia un aprendizaje significativo.

Las actividades lúdicas sean juegos o actividades que les hagan levantar de sus asientos y participar de manera activa según mencionan Núñez Y., Góngora Y., Núñez Yamaisy, Escalante S. (2017), 
ISSN: 2600-5859

"amplían el protagonismo del estudiante en su propio aprendizaje, insertan las posibilidades de la reflexión, el debate y facilitan el desarrollo de habilidades comunicativas". ${ }^{11}$

Así de esta manera los alumnos dejan su rol pasivo y pueden interactuar entre ellos mismos haciendo uso del idioma y utilizando el tema de medicina y su terminología.

Por un lado, el resultado arrojado de la prueba demuestra que la comprensión del tema mejora significativamente con un porcentaje de $89,40 \%$ con el segundo grupo con el enfoque dinámico de las actividades lúdicas y mientras que el primer grupo tuvo un porcentaje como media de $60,78 \%$ con la clase expositiva de preguntas y respuestas.

Adicionalmente, las respuestas de los alumnos en la encuesta mencionan los factores que permiten determinar que el uso de actividades lúdicas tiene un alto impacto en la comprensión del tema en este caso de medicina.

Se determina según menciona Banguela P. (2016) en su estudio sobre actividades lúdicas que los juegos en las clases de medicina en inglés incitan a los alumnos a comunicarse de manera relajada y cooperativa, además ayudan a reducir la tensión de estar en contacto de una lengua extranjera y añade diversión a las clases. Todo esto ayuda en la motivación de los alumnos a participar. ${ }^{3}$

\section{Conclusiones}

- Se concluye entonces de acuerdo a resultados de la investigación que las actividades lúdicas sean juegos, dinámicas o ejercicios activos logran incrementar significativamente la atención, participación y comprensión de los temas médicos tratados en clase debido a su componente entretenido, participativo e integrador que tiene en los alumnos.

- Además, es bien sabido que hay mucha más información en inglés sobre medicina en todo el mundo sean estos avances científicos, papers, casos clínicos y nuevos descubrimientos, pero el recibir las clases con un enfoque lúdico ayuda a los alumnos a comprender mejor y reforzar aquellos temas que encontraran en futuras investigaciones que hagan de los temas.

- Razón imperiosa para que las clases de "inglés para Fines Específicos" como se conoce al inglés técnico por sus siglas en inglés (English for Specific Purposes: ESP) sean dadas con 
ISSN: 2600-5859

este nuevo enfoque donde el alumno se involucre más activamente y su participación sea más plena.

- Finalmente, los alumnos al recibir las clases de medicina en Ingles de manera activa, lúdica y entretenida no solo disfrutarán más las clases, sino que también podrán estar mejor preparados para comunicarse en esta lengua extranjera con otras personas sean pacientes o personal de la salud dentro del país o fuera de él y así puedan ser más competentes en su campo de acción.

\section{Referencias bibliográficas}

Álvarez, V. (2010). EL valor didáctico de la literatura folclórica para enseñar inglés. Pedagogía Magna, 243-250.

Andreu, M., \& García, M. (2014). Actividades lúdicas en la enseñanza de LFE: El juego didáctico. I Congreso Internacional de español para fines específicos, 121-125.

Banguela, P., \& Nodarse, N. (2016). Compendio de actividades lúdicas para enseñar la gramática inglesa en II año de medicina. Revista educación médica del centro-, 8(1). Obtenido de http://www.revedumecentro.sld.cu/index.php/edumc/article/view/581/html_97

Herrell, A., \& Jordan, M. (2012). 50 Strategies for teaching English language learners. Boston: Pearson.

Herrera, J. (2012). Los juegos, una alternativa creativa en la enseñanza de la traumatología. Revista Educación Científica Salud, 7.

Kazarián, Y., \& Prida, M. (2014). Actividades para motivar el aprendizaje de los estudiantes en las clases de inglés. Revista Habanera de Ciencias Médicas, 12.

Labrador Piquer, M., \& Morote, P. (208). El Juego en la enseñanza de ELE. Glosas Didácticas, 84.

Lee, W. (2005). Language Teaching Games. New York: Oxford University Press.

Lightbown, Lightbown, P., \& Spada, N. (2006). How languages are learned. New York: Oxford University Press.

Moré, M. (2008). English Through Medicine I. La Habana: Editorial Ciencias Médicas 
ISSN: 2600-5859

Núñez, Y., Góngora, Y., \& Mayedo, Y. (2017). Set de tareas docentes para darle salida a la estrategia curricular de MNT en el inglés general en las carreras médicas. VI Jornada Científica de la SOCECS, 11.

Ortiz Sánchez, N., Zamora, M., Díaz, B., Vázquez, M., \& Rodríguez, D. (2016). Desarrollo de la Expresión Oral en inglés con fines específicos en la Carrera de Medicina. Humanidades Médicas, 285-300.

Pimienta, S. (2012). Estrategias de Enseñanza Aprendizaje-Docencia universitaria basada en competencias. México: Cámara Nacional de la Industria Editorial Mexicana.

Rosas, Rosas, R., \& Ceric, F. (2015). ¿Pruebas Tradicionales o Evaluación Invisible a través del juego? Nuevas fronteras de la evaluación cognitiva. Psykhe, 24(1), 1-1. doi:10.7764/psykhe.23.2.724

Soto, Y. (2013). La enseñanza lúdica y el aprendizaje del idioma inglés. Juegos para el desarrollo de la expresión oral en los estudiantes de cuarto año de la carrera de Medicina. Revista Médica Electrónica Portales Médicos, 4.

Swain, M., \& Lapkin, S. (1995). Problems in output and the cognitive process they generate: Steps toward second language learning. The Ontario Institute for Studies in Education, 16(3), 371-391.

Vallejos, Á., Latorre, S., \& Ávila, M. (2016). Didácticas innovadoras en el aprendizaje de la farmacología en estudiantes de medicina. Revista del Hospital Italiano de Buenos Aires, 36(3), 99-106

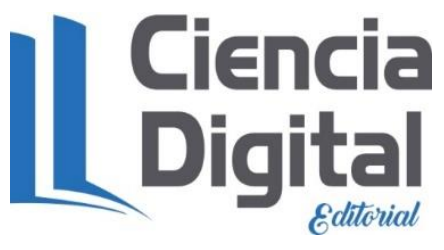


ISSN: 2600-5859

Vol. 3, N¹.1, p. 198-211, febrero, 2020

\section{PARA CITAR EL ARTÍCULO INDEXADO}

Meza Fiallos, C. E. (2020). Manejo de actividades lúdicas con estudiantes de medicina en inglés. ConcienciaDigital, 3(1.1), 194-207. https://doi.org/10.33262/concienciadigital.v3i1.1.1140

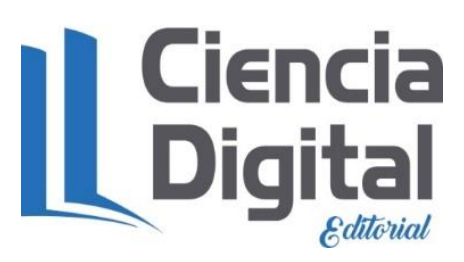

El artículo que se publica es de exclusiva responsabilidad de los autores y no necesariamente reflejan el pensamiento de la Revista Conciencia Digital.

El artículo queda en propiedad de la revista y, por tanto, su publicación parcial y/o total en otro medio tiene que ser autorizado por el director de la Revista Conciencia Digital.
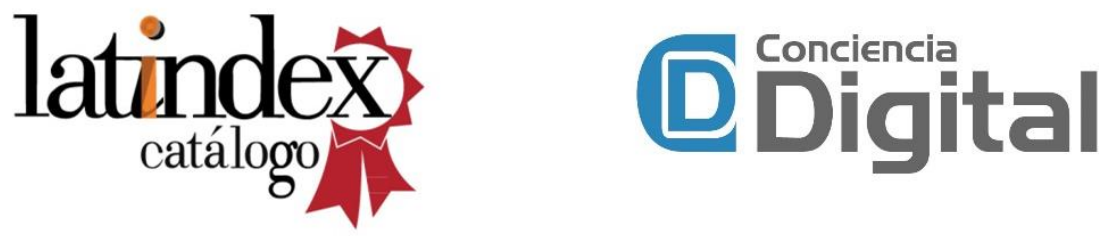